\title{
Assurance de Qualité et GBEA : Généralités et quelques applications à l'analyse en biologie de la reproduction
}

\author{
FERARD G.
}

Groupe de Travail Assurance de Qualité en Biologie de la Reproduction (BLEFCO, CECOS, $S A L F, S F B C$ ) ; Laboratoire de Biochimie Appliquée, Faculté de Pharmacie de Strasbourg,

Université Louis Pasteur, 74 route du Rhin, BP 24, 67401 Illkirch-Graffenstaden

\section{RESUME}

Après avoir défini Qualité, Assurance de Qualité et Contrôles de Qualité, l'auteur traite de l'application des principes généraux de l'Assurance de Qualité à l'ensemble des analyses et observations de Biologie Médicale y compris celles qui concernent la Biologie de la Reproduction. L'importance d'une organisation spécifique des tâches et d'une coordination entre les acteurs participant à la réalisation des tâches entre la prescription et l'envoi d'un compte rendu sont soulignées. L'intérêt de rédiger des procédures écrites, adaptées aux conditions locales de fonctionnement est rappelé de même que la nécessité d'une formation et d'une information suffisante des personnels. Toutes ces informations figurent dans le "Guide de Bonne Exécution des Analyses" ou GBEA publié en décembre 1994.

Mots Clés : Assurance de Qualité ; Contrôles de Qualité; Procédures ; GBEA.

\section{INTRODUCTION}

La démarche Qualité n'est pas d'apparition récente : Dès le moyen âge, les corporations réglementent les différents métiers. Colbert disait déjà "Si nos fabriques imposent, à force de soin, la Qualité supérieure de nos produits, les étrangers trouveront avantage à se fournir en France et leur argent affluera dans le royaume" (3 Août 1664).

Mais c'est avec l'industrialisation que la recherche de la qualité devient une préoccupation constante. Elle a d'abord touché des secteurs stratégiques, à risques, comme le militaire, l'espace, le nucléaire. Puis apparaissent les cercles de qualité. Les laboratoires n'ont pas échappé à cette tendance puisque dès 1965, la Société Française de Biologie Clinique est à l'origine d'un Contrôle de Qualité d'abord en Biochimie puis dans d'autres disciplines telles que la Bactériologie, l'Hématologie, l'Immunologie, la Parasitologie ... Aujourd'hui, l'Assurance de Qualité s'est développée dans le domaine du laboratoire d'analyse de biologie médicale dans divers pays (Belgique, Espagne, France, UK, Pays Bas, Scandinavie, Suisse, USA ...) à l'initiative de sociétés savantes ou d'agences gouvernementales. Le relais a été pris au niveau européen avec des normes EN et au niveau mondial avec celles de l'ISO. L'objet de cette présentation est de définir et de donner les principales caractéristiques des concepts ayant trait à la qualité et d'indiquer quelques applications dans la réalisation de tests de laboratoire concernant la biologie de la reproduction. 


\section{QU'EST-CE QUE L'ASSURANCE DE QUALITE ?}

Par définition, l'Assurance de Qualité telle que définie dans le Guide de Bonne Exécution des Analyses (GBEA) est "l'ensemble des actions préétablies et systématiques nécessaires pour qu'un produit ou un service satisfasse aux exigences de Qualité“.

La définition renvoie à la définition de Qualité :

"Aptitude d'un produit ou d'un service à satisfaire les besoins de l'utilisateur" (Afnor), c'est-à-dire du prescripteur et du patient, ce qui peut aussi être décrit par l'équation suivante :

Qualité $=\frac{\text { Performance fournie }}{\text { Performance attendue }}$

Cette présentation permet de percevoir le caractère subjectif de l'appréciation de la qualité par le "fournisseur" et par le "client" d'où la difficulté d'évaluer la qualité.

L'Assurance de Qualité est une disposition de type organisationnelle qui procède d'une décision a priori à savoir "engager une politique de Qualité". Le caractère organisationnel doit être souligné car dans le domaine des analyses ou des observations de spécimens biologiques, de très nombreux participants et tâches séparent la prescription médicale du rapport final d'analyses ou d'une décision thérapeutique, d'où la nécessité d'une organisation rigoureuse et performante. Ce point apparaît aussi dans le GBEA qui distingue des temps préanalytiques, analytiques et postanalytiques : Plusieurs étapes telles que prélèvements, identification, transport, conservation, traitement préanalytique (coloration par exemple), analyse(s) et observation(s), validation (à l'aide d'un contrôle de Qualité Interne), calcul d'un résultat (Indice d'Anomalies Multiples par exemple), présentation des résultats (standardisés par exemple par rapport aux recommandations OMS), transmission, archivage (mode, délai) sont mentionnées. Le GBEA précise: "la qualité ne dépend pas seulement de l'analyse (de l'observation) mais de l'organisation générale du laboratoire, de la qualification et de la motivation du personnel et du respect des procédures opératoires", en un mot de l'assurance de Qualité.

Le schéma représenté sur la figure 1 décrit (sans doute de manière non exhaustive) une séquence de nombreuses opérations et la participation de différents personnels intervenant entre la prescription et l'envoi d'un compte rendu d'analyses, d'une interprétation, d'un acte thérapeutique. Les tâches, les participants, les maillons critiques, la cohérence intermaillons peuvent changer selon l'analyse et ses conditions locales de réalisation. Mais il y a toujours

\section{Figure 1 : La chaîne des évènements et des participants.}

Prescription Clin.

Prélèvement Clin., Biol.

et informations

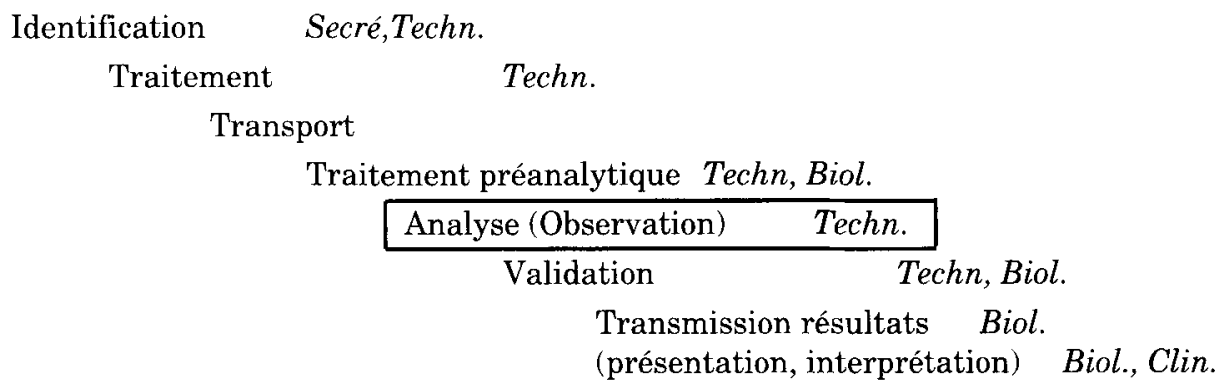


intérêt à simplifier la chaîne de manière à améliorer le transfert d'informations, la coordination entre les acteurs.

Les discussions que nous avons eues dans le cadre du groupe de travail "Assurance de Qualité en Biologie de la Reproduction" ont montré que les principes de l'Assurance de Qualité sont applicables à ce domaine.

La représentation chaîne d'événements indique aussi qu'une optimisation des temps analytiques (ou d'observation microscopique) bien que fondamentale ne suffit pas à garantir la Qualité. Il devient nécessaire d'analyser et de standardiser les différentes étapes depuis le prélèvement jusqu'à la manière de présenter les résultats pour une meilleure efficacité (Tableau 1). Les mots clés apparemment applicables aux analyses concernant la Biologie de la Reproduction sont organisation, formation, transfert d'information.

Tableau 1 : Quelques observations générales à propos de la Qualité.

- La qualité dépend d'une bonne coordination entre les participants (formation, organisation)

- La qualité est d'autant plus difficile à assurer que la chaîne est complexe (organisation)

- Les maillons critiques varient avec le type d'analyse et d'organisation (analyse de la situation locale)

- Le stade analytique ou d'observation n'est qu'une part de la chaîne

- Le stade préanalytique est souvent critique (transfert d'information)

- Le stade postanalytique peut être critique (organisation)

Les domaines généraux concernés sont indiqués en italique

\section{CONTROLES DE QUALITE ET ASSURANCE DE QUALITE}

La démarche Contrôle de Qualité a été et reste essentielle. Par définition, le Contrôle de Qualité comprend l'ensemble des procédures mises en œuvre pour vérifier par des indicateurs le maintien de la Qualité des résultats.

- Le Contrôle de Qualité interne au laboratoire est avant tout une possibilité de validation d'une partie de la chaîne, le plus souvent au niveau analytique. Ce point est abordé dans le GBEA qui parle de validation technique à la suite de la consultation des données du Contrôle de Qualité interne. Dans le domaine du spermogramme, il est envisageable qu'un contrôle ponctuel interne soit organisé et réalisé avec un jeu suffisant de lames "typiques" régulièrement intégrées dans les séries d'analyses. Un tel Contrôle de Qualité interne peut aussi servir à évaluer la cohérence intertechnicien dans le laboratoire et par là définir un niveau de qualité réellement observé dans un laboratoire ou encore un besoin de formation complémentaire. Les performances concernant d'autres étapes peuvent être évaluées de la même manière (coloration, mesure de volumes, comptage ...).

- Le Contrôle de Qualité externe appelé "Evaluation Externe de la Qualité" dans le GBEA est une possibilité de comparaison des résultats d'un laboratoire avec ceux d'autres laboratoires. Il représente aussi un outil de suivi de l'évolution de la qualité et, on l'oublie trop souvent, un moyen de formation des personnels des laboratoires par les commentaires associés au traitement statistique des données.

Le domaine du spermogramme présente la particularité que les matériaux de contrôle, ici les lames, sont stables et qu'elles peuvent ainsi être relues après que le laboratoire ait reçu les commentaires de l'organisme chargé du Contrôle de Qualité externe, ce qui facilite l'utilisation de ces données comme outil de formation (cf l'article d'A. Bourguignat).

L'Assurance de Qualité procède de démarches mises en œuvre a priori. Elles 
comprennent essentiellement la définition d'une Politique de Qualité, la rédaction de procédures (cf plus bas) concernant des tâches générales comme prélèvements, leur identification, locaux, appareillage, techniques de mesure, validation des résultats, formation des personnels, transmission des résultats, documentation des données Qualité. Au contraire, le Contrôle de Qualité pour nécessaire et utile qu'il soit n'implique pas obligatoirement la Qualité. Il permet d'attirer l'attention sur des insuffisances, sur la nécessité de changer de techniques, ou de mieux former le personnel. On notera aussi que nous avons souvent tendance à contrôler les étapes les plus simples, les mieux définies, les plus localisées, les plus proches de nous. Une démarche Assurance de Qualité est complémentaire au Contrôle de Qualité. Pour être pleinement efficace, elle ne doit pas rester interne au laboratoire, mais aussi concerner les étapes extralaboratoires telles que l'information du consultant, le transport et la conservation du prélèvement d'un côté de la chaîne et la présentation, l'interprétation et la transmission du résultat de l'autre côté de la chaîne.

\section{PROCEDURES}

Le terme de procédures correspond à des "instructions écrites, propres à chaque laboratoire, décrivant les opérations à effectuer, les mesures à appliquer dans le laboratoire“. L'avantage de rédiger des procédures est d'éviter des dérives, de rester fiable même lors de changement de personnel. La règle d'or peut être résumée comme suit (Figure 2).

Figure 2: Les 4 étapes intervenant dans l'organisation de la Qualité.

$$
\begin{aligned}
& \text { Ecrire ce que l'on a à faire } \\
& \text { Faire ce qui est écrit } \\
& \text { Documenter ce que l'on a fait } \\
& \text { Réagir s'il y a lieu }
\end{aligned}
$$

Il y a grand intérêt à définir un plan général pour la rédaction des procédures, à les faire circuler pour commentaires, à utiliser l'outil informatique pour les classer et les faire évoluer.

\section{GBEA : CONSEQUENCES}

Le GBEA est un texte réglementaire, et par là contraignant puisqu'il instaure le développement dans les laboratoires d'un système Qualité. Mais il laisse à chacun la liberté de choisir ses méthodes de mesure, réactifs, équipements, modes de formation et préserve ainsi l'évolutivité des laboratoires.

Nous constatons aussi que depuis la parution du GBEA, les relations entre les laboratoires et les Industriels changent dans un sens positif. Ceci est important puisque les producteurs de nouvelles technologies (analyse d'images par ex), d'automates, de nouveaux tests peuvent aider à améliorer la Qualité et l'efficacité des laboratoires non seulement en vendant des produits de qualité, mais aussi en mettant à disposition des produits faciles à utiliser, en transmettant une documentation objective, à visée éducative, en développant des outils permettant le contrôle in situ des machines souvent très complexes qui nous sont proposées, en organisant des stages de formation.

\section{MISE EN EUVRE DU GBEA}

Est-ce que cette démarche Qualité est adaptée aux centres de recherche en biologie médicale?

La réponse est sans doute oui puisque les résultats des études qui ne sont pas suffisamment solides en terme d'assurance de qualité sont souvent refusées dans les revues scientifiques. Cette remarque concerne tout particulièrement les études épidémiologiques.

Qu'elle est la taille minimum d'application d'une démarche Qualité? 
Il est vraisemblable qu'un laboratoire qui n'emploierait qu'une personne pourrait développer une démarche Assurance de Qualité. La nomination d'un responsable Qualité, imposée par le GBEA, n'exclut pas que le directeur du laboratoire soit aussi responsable Qualité.

Enfin, il est utopique d'imaginer que la constitution d'un simple catalogue de procédures soit suffisant pour obtenir de manière durable un niveau de qualité donné. L'adhésion de l'ensemble du personnel, la mise à disposition des procédures ainsi que leur mise à jour sont au moins aussi nécessaires. Inversement, il est souhaitable de ne pas développer de système Qualité trop rigide et complexe (Tableau 2)

Tableau 2 : Quelques causes d'échecs d'une démarche Qualité

- Formation insuffisante des personnels

- Responsabilités non définies

- Informations insuffisantes ou perdues (écrits)

- Inadéquation des moyens (y compris formation)

- Complexité trop grande du système Qualité

- Trop grande "impatience" à vouloir introduire une démarche Qualité

Remarque : L'échec n'est pas forcément lié à la taille du laboratoire

\section{CONCLUSIONS}

En conclusion, il semble indispensable et profitable de développer des démarches dynamiques d'Assurance de Qualité, intégrant l'ensemble des personnels des laboratoires, préservant leur autonomie, leur savoir faire pour la satisfaction des prescripteurs et des demandeurs d'examens. Cette stratégie, devrait prendre en compte non seulement les tâches et les acteurs depuis la prescription jusqu'au résultat d'analyse, mais encore les besoins des demandeurs, d'où une nécessité d'un meilleur échange d'informations. On peut ainsi espérer que la cohérence interlaboratoire des résultats et donc leur efficacité soit améliorée. Cette démarche qui se généralise est en France définie par les obligations et recommandations qui figurent dans le GBEA, ce qui signifie que ce texte est le référentiel qualité dans notre pays.

\section{REFERENCE}

1. Guide DE BonNe EXECUTION DES ANALYSES DE BIOLOGIE MEDICALE. Arrêté du 2 novembre 1994. Journal officiel de la République française du 14 décembre 1994 : 17193-201.

\section{ABSTRACT}

Quality Insurance in Analysis Concerning Biology of Reproduction

\section{G. FERARD}

Definitions of Quality, Quality insurance and Quality controls are given. The applicability of general principles of Quality assurance to the analysis concerning the Biology of the Reproduction is underlined. The necessity of an overall organisation taking into account the different participants and services is stressed. The model with preanalytical, analytical and postanalytical steps is described. Good coordination between the different actors along the chain of interdependent steps is crucial. Documentation is an essential part of a quality system. All these elements and recommandations are indicated in the "Guide de Bonne Exécution des Analyses" or GBEA which was published in december 1994 and which is the french reference for the implementation of a quality system in the laboratories.

Key words : Quality insurance, Quality controls. 